\title{
Automated Guidance System Drogue to Probe Tanker During in-Flight Refueling
}

\author{
M.K. Filyashkin \\ National Aviation University Aviation \\ Computer-Integrated Complexes Department \\ filnik@ukr.net
}

\begin{abstract}
Here are considered benefits of airlift using a phase in-flight refueling civil aircraft. Such technologies provide in particular for regional aircraft their main advantages at long flights. The variants of automation of this phase of the flight are proposed.
\end{abstract}

Keywords-refueling; probe of refueling; drogue; refueled aircraft; tanker aircraft; control laws; remote-controlled drogue

\section{INTRODUCTION}

To optimize the delivery of passengers is requiring for a particular flight not only choose the type of vehicle, but also ensure the effectiveness of its use by applying the modern transport technologies. Improving economic efficiency of air transport services can be accomplished through the use for regional aircraft technology of in-flight refueling (IFR), which can provide the exploitation of these aircraft on long-haul nonstop flights instead of expensive long-haul aircraft [1].

A striking example of regional jets, for which can be applied, these technologies are modern jets: An-158 (passenger variant) and An-178 (transport aircraft) of the company Antonov. Comparative analysis of the technical characteristics of the aircraft An-158, characteristics of the aircraft of medium-range Boeing 737-900 and the long-range aircraft A330-300 of the company Airbus shows that short-haul (SHA) and medium-haul aircraft (MHA) can well solve the problem of transportation of passengers on long international lines. In this case the characteristics of navigation equipment of the aircrafts approximately the same and a flight comfort on SHA and on MHA is not worse than on the elite long-haul mainline jets (LHA). The problem of LHA is also the fullness of load flights, which is low for the long international lines.

The technology of IFR can significantly reduce the fuel consumption, will allow several times to increase the commercial aircraft load and reduce the emissions of $\mathrm{CO}^{2}$, as well as reduce the cost of the flight fleet. In addition, the implementation of direct long-haul flights using regional jets proportion of flights with a stopover in the airline hub is reduced, because passengers can be delivered directly to the destination without change of flights in international airports.

All this emphasizes the urgency of developing technologies of IFR for civil aircraft. The development of this idea is currently engaged in leading aviation research organizations in Europe in the framework of "Recreate" project [2], as well as scientific institutions of Russia and Israel.

\section{Problem Statement}

Analysis of existing refueling systems shows that the most common and relatively simple system is the "probe-and-drogue system". Therefore this method is taken as the basis for the development of the automated system of IFR for civil aircraft.

But in order to reduce the requirements for pilots of civil airliners invited to use for the "probe-and-drogue» system the method of "reverse IFR". This method requires no special training pilots of the aircraft, which should be re-filled. The crew of this aircraft, equipped with an unified unit of refueling, by command of the tanker only let out a flexible hose with the drogue, which trails from the aircraft. The entire alignment process of rapprochement and contact performs a trained crew of the tanker aircraft, which is approaching to the commercial aircraft behind and performing the maneuvers, connects its own fueling system with the extended drogue. Alterations in accordance with the "inverse IFR" expose only the unit of refueling suspended under the fuselage.

Additionally refueling process can be automated to reduce the load on the tanker aircraft crew. At the same time it's necessary to automate not only the control of the aircraft, as well as the control of a drogue of IFR, equipping the drogue aerodynamic control surfaces and thereby turning it into the remote-controlled UAV, which the crew of tanker will be able to direct on its probe of a refueling system.

The control system of a drogue using their own control arrangements can carry out the stabilization of a drogue in the air, fending turbulent perturbation, and as well as to direct the drogue on the probe of a tanker aircraft in an automatic or automated regime of control.

The statement of the problem can be formulated as follows: for the parry of turbulent perturbations acting on the drogue and impeding the contact process, on the basis of one embodiment of an aerodynamic control of the drogue design the system of its stabilization (SSD), and then based on SSD build the system guidance of the drogue (SGD) at the probe of refueling.

\section{PROBlEM SOLUTION}

In this paper, on an analysis of possible of control methods of a drogue have been selected the system, which is based of changes of flow around opposite sides of the fairing of a drogue. The control system (Fig. 1) uses to change the flow of opposite sides of the fairing the apertures 2 on the front part of the casing 1 of a drogue. Apertures may be closed or opened 
by means of plates connected to the actuator. The apertures have different diameters, so that different combinations of opening and closing of these holes change the overall area of the slot in the casing filling drogue. As actuators are used low-power solenoids. Using this technology changes a speed and a pressure flowing stream along an aerodynamic profile of the drogue and thereby changes the distribution of aerodynamic forces acting on the drogue, that is, to control a movement of the drogue in an air stream.

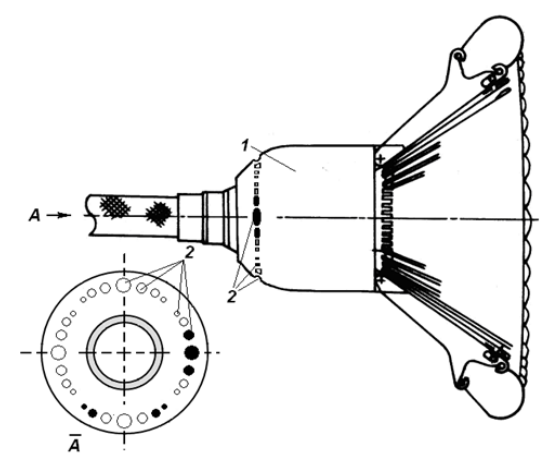

Fig. 1. Aerodynamic control units of a drogue.

To parry disturbances acting on the drogue while refueling in the air, it is proposed the system SSD, a sensor of which is a small, ultralow power, 3-axis digital MEMS accelerometer, installed under the hood of a drogue. The measuring axis accelerometer coincide with an axis of symmetry of the drogue. Outputs of accelerometer are connected to two voltage converters in combinations of open and closed holes. Outputs of the transducers are connected to solenoid valves, which overlap the openings in the fairing of the drogue. The algorithm of the SSD is a classic damper.

Control laws of actuators dampers for vertical and horizontal channels are identical.

$$
\delta_{h}=\left(K_{n_{y}}+K_{\tilde{n}_{y}} / p\right) \Delta n_{y} ; \quad \delta_{z}=\left(K_{n_{z}}+K_{\tilde{n}_{z}} / p\right) \Delta n_{z},
$$

here $\delta_{h}, \delta_{z}$ is travels of dampers in vertical and horizontal channels respectively; $\Delta n_{y}, \Delta n_{z}$ are values of surplus vertical and horizontal accelerations, that are measures ADXL345.

A basis for the system SGD is the technology RTK (Real Time Kinematic) [4], which provides a measure of relative provisions of objects with an accuracy of about $10 \mathrm{~mm}$ at baseline distances of $\sim 20 \mathrm{~m}$.

On the drogue of a suspended refueling aggregate are arranged (Fig. 2) the receiver GPS, the command radio line transceiver, the linear notifier of an entrance of the probe in a basket of the drogue and SSD.

The equipment of a tanker aircraft includes a telescopic probe with the GPS receiver and the webcam, the command radio line transceiver, the joystick remote control of a drogue of co-pilot, the indicator screen and the calculator of SGD. Information from the calculator SGD also direct in the autothrottle of a tanker aircraft. The autothrottle working at the control law (1) implements the exponential trajectory change a speed of docking approach $\Delta V_{3}$, which is proportional to the relative distance of the drogue $\Delta d$, which is calculated on the technology Moving Base RTK, using information two receivers GPS.

$$
p \delta_{\text {throt }}=K_{\dot{V}} p V+K_{V}\left[\left(V_{\mathrm{des}}+\Delta V_{\mathrm{des}}\right)-V\right]
$$

here $\delta_{\text {throt }}$ is the travel of throttle lever; $V_{\text {des }}$ is the desired speed of flight at the phase of refueling; $\Delta V_{\text {des }}=-\Delta d / \tilde{T}$ the change desired speed of approach; $T$ the time constant of exponent.

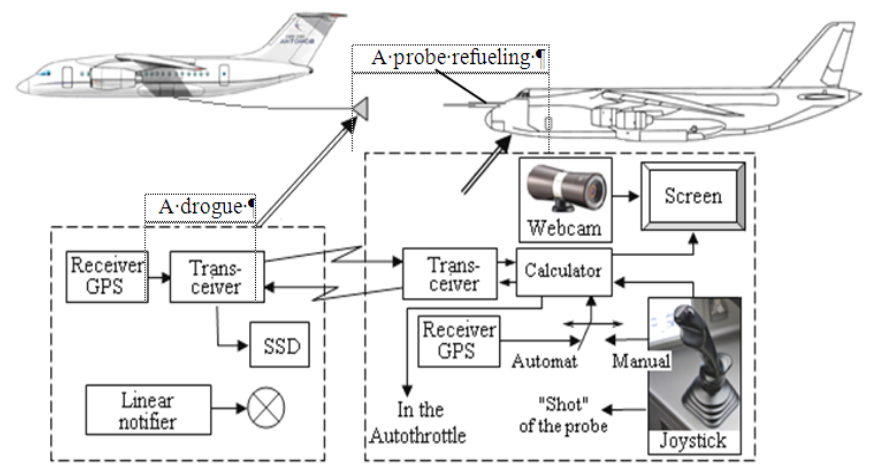

Fig. 2. The guidance system a drogue to the probe refueling.

However, the use of such a law greatly increases time of approach, because direct contact a probe with the drogue theoretically will be realized only in infinity. To avoid this, the asymptote of the exponential must be located behind a drogue at a distance $\Delta d_{\mathrm{as}}$. In this case, the trajectory of an approach is realized in the form of:

$$
\Delta V_{\mathrm{d}}=-\left(\Delta d+\Delta d_{\mathrm{as}}\right) / T
$$

Control laws of actuators of the drogue at the stage of automatic guidance on the refueling probe are of the form:

$\delta_{h}=\left(K_{n_{y}}+K_{\tilde{n}_{y}} / p\right) \Delta n_{y}+K_{h} \Delta h ; \delta_{z}=\left(K_{n_{z}}+K_{\tilde{n}_{z}} / p\right) \Delta n_{z}+K_{z} \Delta z$, here $\Delta h, \Delta z$ is the vertical and lateral deviation of the drogue from the axis of the refueling probe, which are calculated similarly to the calculation of the distance $\Delta d$.

In automatic mode, the control signals $\Delta h, \Delta z$ in control laws are replaced with the signals from the joystick remote control co-pilot, which are received by the command radio line with tanker aircraft.

The research of the proposed loop stabilization drogue conducted by mathematical simulation using the program Simulink, which is part of the mathematical package Matlab programming.

In researches the model of the dynamic of drogue was introduced as a system of linear differential equations, 


$$
\begin{aligned}
& a_{\ddot{h}} \Delta \ddot{h}+a_{\dot{h}} \Delta \dot{h}+a_{h} \Delta h+a_{\delta_{h}} \Delta \delta_{h}+h_{\text {tur }}(t) \\
& a_{\ddot{z}} \Delta \ddot{z}+a_{\dot{z}} \Delta \dot{z}+a_{z} \Delta z+a_{\delta_{z}} \Delta \delta_{z}+z_{\text {tur }}(t) .
\end{aligned}
$$

The mathematical model of the drogue motion dynamics was obtained by simplification the known model motion of hose points [3]:

$$
\rho \frac{\partial^{2} u}{\partial t^{2}}=F \frac{\partial^{2} u}{\partial x^{2}}-\rho g-D \frac{\partial u}{\partial t}-A \frac{\partial u}{\partial x}+A \alpha_{\mathrm{tur}}(x, t)
$$

and given boundary conditions on the right and left ends of the hose respectively:

$M_{\mathrm{dr}} \frac{\partial^{2} u}{\partial t^{2}}=-M_{\mathrm{dr}} g-D_{\mathrm{dr}} \frac{\partial u}{\partial t}-\left(C_{y}+F\right) \frac{\partial u}{\partial x}+\left.C_{y} \alpha_{\mathrm{tur}}(x, t)\right|_{x=L}$, $\left.u(0, t)\right|_{x=0} \cdot$

where $u,(\Delta h, \Delta z)$ are functions of coordinate deviation of hose points (drogue); $\Delta \delta_{h}, \Delta \delta_{z}$ are impacts of the drogue control system; $M_{\mathrm{dr}}$ is the mass drogue; $\rho$ is the linear density of the hose, $t$ is the time; $g$ is the acceleration of gravity; $D$ is the damping coefficient of antihunting; $D_{\mathrm{dr}}$ is the damping coefficient the drogue; $A$ is the coefficient of the linear aerodynamic force; $F$ is the force of a hose tension; $\alpha_{\text {tur }}(x, t), h_{\text {tur }}(t), z_{\text {tur }}(t)$ are components of gusts turbulent atmosphere; $L$ is the hose length; $C_{v}$ is the aerodynamic coefficient of the drogue; $x$ is the coordinate along the hose; $a_{\ddot{h}}, a_{\dot{h}}, a_{h}, a_{\delta_{h}}, a_{\ddot{z}}, a_{\dot{z}}, a_{z}, a_{\delta_{z}}$ are coefficients of the simplified model of a spatial movement of the drogue.

And at the simulation of a turbulent atmosphere was used the generally recognized Dryden model.

$$
S_{w}(\omega)=\sigma_{u}^{2} \frac{L}{2 \pi V}\left[1+3\left(\frac{L}{V}\right)^{2} \omega^{2}\right] \cdot\left[1+\left(\frac{L}{V}\right)^{2} \omega^{2}\right]^{-2}
$$

where $\sigma_{u} \quad 1 \sigma$ the mean-square value of the speed of a random eddy wind; $L$ is the linear scale of an atmospheric turbulence; $V$ is the flight speed.

In researches of dynamics of movement in an air the components of a spectral density of an atmospheric turbulence can be extracted from a random noise by means of the shaping filter

$$
\Phi_{w}(p)=1 /\left(T_{\mathrm{f}} p+1\right)
$$

Simulation results (Figs 3 and 4) showed the efficiency of developed algorithms SSD and SGD, even when refueling in a turbulent atmosphere. In particular, the amplitude of bumpiness of the drogue in a turbulent flow is reduced by almost four times. Significantly reduces the docking time and simplifies the process of piloting a tanker aircraft. The pilot for 5-7 s eliminates the error of guidance and to further easily holds a drogue in the region of the contact zone.

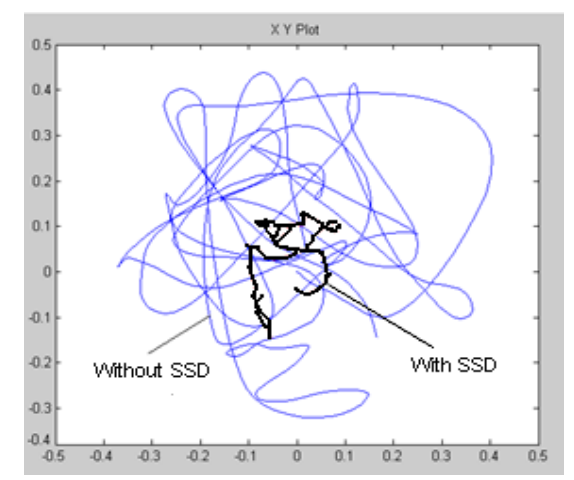

Fig. 3. Simulation results of the drogue motion at refueling in the turbulent atmosphere.

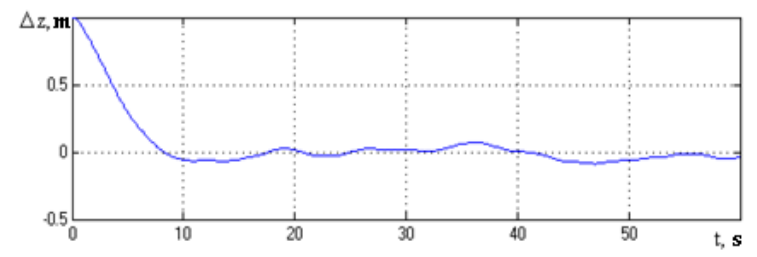

Fig. 4. Simulation results of the guidance a drogue at the probe refueling.

Given the unreliability of the GPS navigation in the last stage of guidance is recommended to switch at automated mode by using the joystick, and the position of the drogue relative to the probe be evaluated visually using a video screen. In the channel of rapprochement, can be used the information from the high-precision distance measuring sensors, such as optical or ultrasound.

Implementation of the proposed AAR system will improve safety at the stage of contacting and significantly reduce the time of refueling.

\section{CONCLUSIONS}

The technology in-flight refueling of regional jets will allow: to increase their commercial payload; significantly save fuel and reduce $\mathrm{CO}^{2}$ emissions into an atmosphere.

The proposed systems SSD and SGD greatly facilitates the piloting technique at the contacting stage, even when refueling in a turbulent atmosphere, and substantially reduces psychophysical loads of pilots.

\section{REFERENCES}

[1] Velez Ukrainian aviation company-Velez "Passenger air traffic". http://velesavia.com/passajirskie-aviaperevozki.html.

[2] Project - RECREATE. http://www.cruiser-feeder.eu/project

[3] G. Obolensky, V. Pohvalensky, and D. Cheglakov, "Algorithm of automatic control aircraft during in-flight refueling." Electronic journal «Proceedings of MAI». Issue no. 65. http://www.mai.ru/

[4] Determination of mutual position of objects by the GPS-measurements. // http://www.eftgroup. ru/ work /seasurve 